\title{
Resveratrol chemoprotective effect and COX 2 down regulation in tumor suppression in DMBA induced breast cancer in female Sprague Dawley rats
}

\author{
Aneena Suresh ${ }^{1 *}$, Rajat Rana ${ }^{2}$, Keerthana C. ${ }^{1}$
}

\begin{abstract}
${ }^{1}$ Department of Pharmacy
Practice, JSS College of

Pharmacy, Ooty, Tamil Nadu, India

${ }^{2}$ Department of Pharmacy

Practice, Asmara College of

Health Sciences, Asmara,

Eritrea
\end{abstract}

Received: 11 April 2017

Accepted: 06 May 2017

*Correspondence to:

Dr. Aneena Suresh,

Email: aneena@jssuni.edu.in

Copyright: (C) the author(s), publisher and licensee Medip Academy. This is an openaccess article distributed under the terms of the Creative Commons Attribution NonCommercial License, which permits unrestricted noncommercial use, distribution, and reproduction in any medium, provided the original work is properly cited.

\begin{abstract}
Background: Aim of the study was to assess the Chemo protective role of Resveratrol in 7,12-Dimethylbenzanthracene (DMBA) induced breast cancer in Female Sprague Dawley rats and its possible role in down regulation of COX 2, an enzyme known to be expressed in breast cancer tissues.

Methods: A total of 40 female Sprague dawley rats (total 4 groups, $n=10$ per group) 6 weeks old, group 1 on pulverized rodent diet, group 2 DMBA with diet, group 3 DMBA and diet with Resveratrol 100mcg, group 4 DMBA and diet with Resveratrol $200 \mathrm{mcg}$. After 120 days experiment was terminated and tumors were analyzed for multiplicity, incidence and histology. Cox 2 expression was analyzed by Western blot analysis. Values were statistically tested using one way variance and Tukey's comparison test.

Results: Body weight and tumor volume was similar, there was remarkable high latency period for tumor onset and reduction in tumor multiplicity and incidence in resveratrol treated groups. Tumor incidence was $42.27 \pm 10.17$ for Group 2, 21.91 \pm 5.87 for Group 3, 13.73 \pm 3.98 for group 4. Tumor multiplicity was reported as $0.8909 \pm 0.30$ for group 2, $0.1036 \pm 0.04$ for group 3, $0.04545 \pm 0.02$ for group 4 . Histopathological analysis revealed ductal carcinoma in group 2, minor tissue necrosis in group 3 and fibroadenoma in group 4.

Conclusions: Resveratrol has chemoprevention action against DMBA induced breast cancer and suppresses COX 2 expression in breast carcinoma.
\end{abstract}

Keywords: Cox 2, Fibroadenoma, Latency, Resveratrol, Tumor incidence

\section{INTRODUCTION}

Several nonnutritive phytochemicals found in natural products and accompanying pharmacological aspects reveal proof that they inhibit, delay, and/or reverse cancer aroused by either environmental insults and/or lifestyle modifications. ${ }^{1,2}$ Several of these agents act at initiation, promotion, and/or progression stages and are linked with the ontogeny of multistage carcinogenesis.

Resveratrol (trans-3,5,4'-trihydroxystilbene;), an essential element of grapes, berries and peanuts has risen as an "age-prolongation factor". 3 Subsequently, the compound was considered a phytoestrogen due to its powerful estrogenic and even super estrogenic properties. ${ }^{4}$ Resveratrol a stilbene found in the diet of humans was identified as a possible chemopreventiveagent. ${ }^{5}$ Most researches directed on resveratrol during last two decades exposes the mechanisms by which resveratrol's chemo protective effects like reduction of cancer initiation, promotion, progression, as well as reduction of inflammation were testified. ${ }^{6-9}$ In recent years the research proved the anti-cancer property in female rats by enhancing maturation of the mammary gland, reducing 
cellular proliferation, and increasing apoptosis in mammary epithelial cells. ${ }^{10}$

The present study was conducted to evaluate the effect of resveratrol to down regulate cox-2 which in turn will inhibit 7,12-dimethyl benzaanthracene (DMBA) induced tumorigenesis in rat model. We also investigated the dose dependent therapeutic effect of resveratrol. Previous studies have confirmed the role of transcription factor kappa b2 in promoting tumor metastasis by binding to various promoter regions of targeted genes such as c-myc and p53. ${ }^{11}$ This a first study to measure anticancer property of resveratrol at varied doses in vivo. The study analyzed the association between resveratrol and cox-2 down regulation, an enzyme known to induce nitric acid synthase, recognized to potentiate critical phases in proliferation, metastasis, angiogenesis and apoptosis of cells. ${ }^{12,13}$ The study was carried out with the hypothesis that resveratrol has Anti-cancer property and causes down regulation of COX-2.

\section{METHODS}

DMBA of highest purity was purchased from sigma chemical co. (St. Lewis Mo, USA), Resveratrol from (Alexis cooperation, CA). All other chemicals used were of high analytical grade.

\section{Animal and experimental design}

Female spray dawley rats $(n=40)$ were provided by experimental animal house, PSG Institute of medical sciences and research, Tamilnadu. All the rats arrived at the age of 40 days and were placed on a standard vitamin enriched diet consisting of $60 \%$ roasted Bengal gram powder, $23 \%$ wheat flour, $5 \%$ skimmed milk powder, $4 \%$ casein, $4 \%$ refined oil, salt mixture with $4 \%$ starch and choline. The animals were housed 3 per cage in a standard well ventilated wire bottomed steel cages, maintained under hygienic conditions with proper aeration at $25 \pm 2^{\circ} \mathrm{C}$ and a relative humidity of $45-50 \%$ under $12 \mathrm{hr}$. light and darkness cycle. The standard procedures were followed in accordance with Indian national law on animal care and use for maintaining the experimental animals\& ethically approved procedures. All the rats were completely inspected for any major lesions, pathogenic infections including complete determination of health status.

After arrival in the labs the rats were acclimatized on pulverized diet for 3 days. Based upon their body weight all the rats were divided in to three groups: group $1(\mathrm{n}=$ $10)$, received pulverized rodent diet, labeled as negative control; group $2(\mathrm{n}=10)$ received DMBA with pulverized diet; group $3(n=10)$ received DMBA with pulverized diet containing resveratrol $(100 \mathrm{mcg} / \mathrm{rat})$; and group $4(\mathrm{n}=10)$ received DMBA along with experimental pulverized diet containing resveratrol $(200 \mathrm{mcg} / \mathrm{rat})$.

\section{Experimental food}

Free access to diet and drinking water were allowed to the animals throughout the study period. The dose was decided based upon the average food intake by the rats which was around 13-18g/rat/day. This study evaluated dose of resveratrol at dose of $100 \mathrm{mcg} / \mathrm{rat}$ and higher dose of $200 \mathrm{mcg} / \mathrm{rat}$.

A stock solution of resveratrol was made in dimethyl sulfoxide (DMSO) at concentration of $10 \mathrm{mM}$. Resveratrol dissolved in $70 \%$ ethanol was added to the standard experimental pulverized diet in one day advanced and left for room temperature for one day. The food was given to the rats after ethanol evaporation.

\section{Study design}

The study design of this experiment was stimulated from the study design used by Banerjee et. al. ${ }^{14}$ The rats belonging to appropriate experimental group received resveratrol beginning at day 45 of age. After one week (day0) the rats belonging to group 2, 3 and 4 were given sub optimal dose $(10 \mathrm{mg})$ of DMBA by gavage in sesame oil. To evaluate the carcinogenic end points suboptimal dose of DMBA was administered. Once a week all the experimental rats were weighed, palpated for mammary tumours. After 120 days the study was terminated and all the animals including group 1, the animals that didn't developed tumours were sacrificed by $\mathrm{CO}_{2}$ asphyxiation. After the sacrifice the rats were incised and the tumours were removed and fixed in $10 \%$ buffered formalin. The tumours were evaluated for histopathology and were graded either as fibro adenoma or carcinoma. The specific end points for analysis included; the number of animals with tumours (tumour incidence); the number of tumours per animal (tumour multiplicity); tumour latency period; and tumour volume determined by formula $\mathrm{V}=4 / 3 \pi \mathrm{r} 3$, where $\mathrm{r}$ is half of the average diameter measured with a vernier calipers at two different planes (in millimeters).

\section{Tumour histology}

At 3 to $5 \mu \mathrm{M}$ paraffin embedded, formalin fixed tissues were sectioned and stained with Hematoxylin-eosin $(\mathrm{H}$ and E) (Sigma Co Ltd. USA) observed with light microscope (Olympus, USA). The end points evaluated the sections were growth pattern tumour cell cytology and cystic change.

\section{Western Blot analysis}

Mammary tumour specimens were collected from each group and homogenized at $4^{\circ} \mathrm{C}$ using Douncehomogenizer in RIPA (RadioImmunoprecipitation assay) lysis buffer for extracting total cell protein. On 10\% SDS-PAGE gel (Sodium Dodecyl sulfate-Polyacrylamide gel electrophoresis) $60 \mathrm{mcg}$ of whole cell protein was resolved. Then the 
protein was shifted to a nitrocellulose membrane blocked with 5\% non-fat milk and probed with antibody against COX 2 (1: 1000). After washing the blots they were reacted with horse radish peroxidase-conjugated secondary antibodies for $1 \mathrm{hr}$. and finally detected by ECL reagent (Sigma Co Ltd. USA).

\section{Statistical analysis}

The final end points analyzed were body weight, tumour incidence, tumour multiplicity and tumour volume and for all the groups. This study also evaluated role of Resveratrol in COX 2 down regulation. The data was recorded, analyzed and compared using one way analysis of variance using Graphpad prism 5.3.

\section{RESULTS}

\section{Food intake and body weight}

To determine the Chemo protective effect of resveratrol the experiment was designed to evaluate the preventive effect of continuous dietary administration beginning 1 week before DMBA administration until the experiment was terminated. No significant differences were detected in food intake of the rats under all groups. Table 1 shows the mean values of various parameters regarding DMBA induced tumor in animals.

Table 1: Effect of resveratrol and 7, 12 dimethyl Benzanthracene (DMBA) on parameters of control and experimental groups.

\begin{tabular}{|ll|lll|}
\hline \hline Parameters & G1 & G2 & G3 & G4 \\
\hline Diet & $\begin{array}{l}\text { Pulverized } \\
\text { rodent diet }\end{array}$ & $\begin{array}{l}\text { DMBA+ } \\
\text { Pulverized } \\
\text { rodent diet }\end{array}$ & $\begin{array}{l}\text { DMBA + Pulverized } \\
\text { rodent diet + Resveratrol } \\
(100 \mathrm{mcg})\end{array}$ & $\begin{array}{l}\text { DMBA + Pulverized } \\
\text { rodent diet + Resveratrol (200 } \\
\text { mcg) }\end{array}$ \\
\hline Body weight (gms) & $230.6 \pm 7.36$ & $228.2 \pm 7.19$ & $224.2 \pm 6.74$ & $221.2 \pm 6.52$ \\
\hline Tumor Incidence & 0 & $42.27 \pm 10.17$ & $21.91 \pm 5.87$ & $13.73 \pm 3.98$ \\
\hline Tumor Multiplicity & 0 & $0.8909 \pm 0.30$ & $0.1036 \pm 0.04$ & $0.04545 \pm 0.02$ \\
\hline Tumor Volume & 0 & $1.69 \pm 0.42$ & $1.36 \pm 0.27$ & $1.34 \pm 0.24$ \\
\hline
\end{tabular}

*Data are presented as the means \pm standard deviation of four rats from respective group

The average body weight was virtually comparable in all the groups throughout the experiment (230.6 \pm 7.3 , $228.2 \pm 7.2,224.2 \pm 6.7,221.2 \pm 6.5 \mathrm{gms}$ ) for groups $1,2,3$ and 4 respectively; $p=$ non-significant. Also no major adverse effects on weight were observed during the study (Figure 1).

\section{Tumour incidence}

There was no incidence of tumour in group 1 (negative control group), in group 2 at the end of 13 week, $70 \%$ of rats treated with DMBA developed palpable breast tumours, whereas in group 3 which received resveratrol (100mcg) supplemented diet along with DMBA the tumour incidence was $36 \%$ at the end of 13 week.

In Group 4 (200mcg Resveratrol) the tumour incidence was $29 \%$ at the end of 13 week. Tukes Multiple Comparison Testrevealed statistically significant difference between Group 2 and Group 4 although the difference between Group2 and Group3 was statistically not significant (Figure 1).

\section{Tumour multiplicity}

The average number of tumours per tumour bearing animal was $0.89 \pm 0.30 \quad($ mean \pm SE), $0.10 \pm 0.04$ and $0.05 \pm 0.02$ for Group 2, 3 and 4 respectively. On performing Tukes multiple comparison test a statistically significant difference was found in Group 2 vs. Group 3 and group 2 vs. Group $4(\mathrm{p}<0.05)$. Although the difference in Group 3 and Group 4 was not statistically significant but noteworthy in relation to dose (Figure 1).
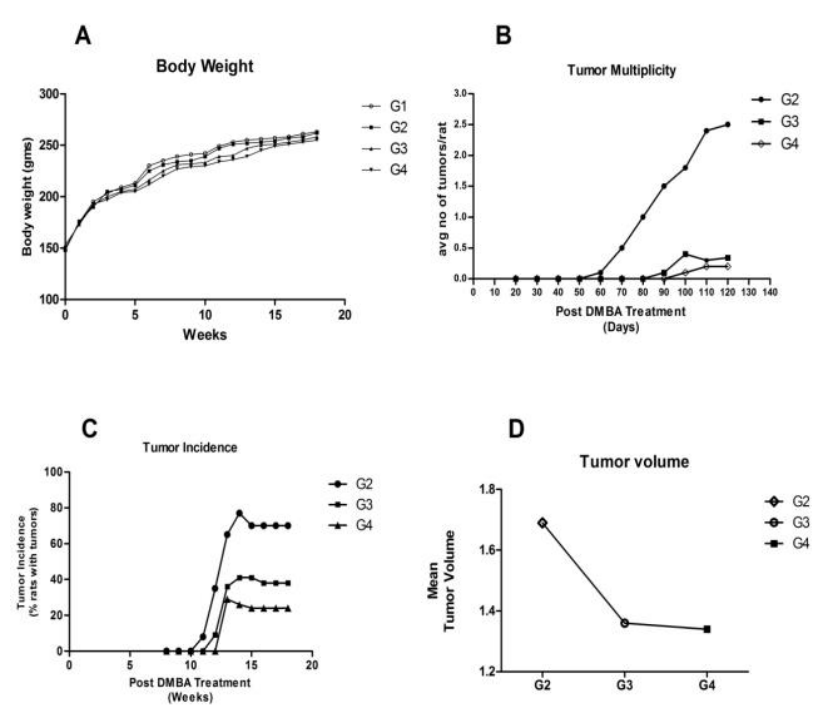

A) The figure shows the average weekly body weights in all groups of female Sprague Dawley rats, B) The figure shows tumor multiplicity of DMBA-induced mammary tumors, C)This figure shows progressive percentage incidence of cumulative palpable mammary tumors, as a function of time after carcinogen treatment, D) The figure shows the average tumor volume in different groups of female Sprague Dawley rats.

Figure 1: Effect of resveratrol on the DMBAinduced tumors. 
Tumour latency period observed in Group 2 was 60 days, 80 days for Group 3 and 102 for Group 4.

\section{Tumour volume}

For examination of tumour volume the tumours were obtained after incising the rats and were measured using the formula $v=4 / 3 \pi r 3$ where $r$ is half of the average diameter measured with a vernier caliper at two different planes. There was no statistical difference between Group $2,3,4$ and the recordings were $(1.86 \pm 0.28$ versus $1.23 \pm 0.34$ verses $1.09 \pm 0.21$ ) respectively (Figure 1 ).

\section{Tumour histology}

The paraffin embedded tissues, formalin fixed were histologically analyzed with H\&E stain at 3 to $5 \mu \mathrm{M}$ section. There was no pathological abnormality in group 1 (negative control). DMBA treated rats (Group 2) developed slight focal micro invasion and ductal carcinoma. In group 3 (DMBA+ resveratrol $100 \mathrm{mcg}$ ) minor tissue necrosis and group 4 (DMBA+ resveratrol $200 \mathrm{mcg}$ ) fibroadenoma was observed. The histology of adenoma was remarkable in group 4 when compared to group 3 (Figure 2).

A

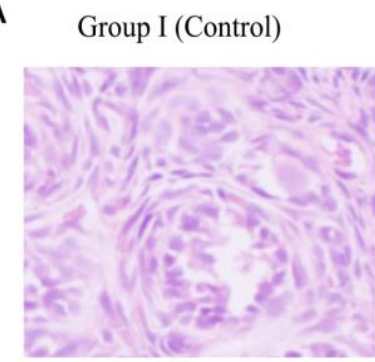

C Group $3(\mathrm{DMBA}+$ Resveratrol $100 \mathrm{mcg}$

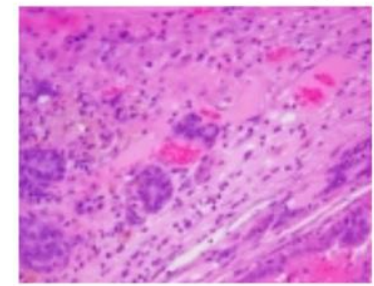

A) In untreated control group normal pathology is observed, B) In DMBA-treated rats ductal carcinoma is observed, C) In DMBA and resveratrol-treated $(100 \mathrm{mcg})$ rats minor tissue necrosis is observed, D) In DMBA and resveratrol-treated $(200 \mathrm{mcg})$ rats fibroadenoma is observed

Figure 2: Effect of resveratrol on the DMBA-induced tumors pathology.

\section{DISCUSSION}

The present study demonstrates significant prophylactic action of Resveratrol in DMBA induced breast cancer in Dawley female rats. In this interventional study Resveratrol was given along with rodent diet as Chemo protective agent before inducing breast cancer, we found that Resveratrol fed Dawley female rats has longer latency period for developing tumours and the tumour multiplicity was higher in DMBA induced cancer group. Whereas Group 3 and 4 which were fed on resveratrol rodent diet developed comparatively less tumours. Also the latency to onset of tumour development was prolonged in resveratrol fed group.

Many previous studies have confirmed the role of resveratrol in inhibiting the breast cancer, the protective effect of resveratrol, since it acts as putative estrogen agonist in differentiation proliferation and maturation of tumour cells. ${ }^{15,16}$ We also report in this study that Resveratrol given before onset of puberty can cause formalin of refractory cell phenotypes by enhancing the differentiation in mammary tissues. We also observed the higher dose of resveratrol is more effective in prevention of tumors onset. Thisanti-cancer property of resveratrol can be attributed to its similar structure acting as partial estrogen receptor. ${ }^{17} \mathrm{We}$ found that resveratrol is having significant role in suppressing COX 2 expressions which explains its tumour suppression property. We report that COX 2 is expressed considerably high in breast cancer tissues and can be regarded as a potential biomarker of breast carcinoma, in addition it was observed that resveratrol doesn't affect the weight gain or have other side effects in rats at high doses. The tumour incidence was least in rats being treated with resveratrol $200 \mathrm{mcg}$ whereas the DMBA group elicited very high tumours incidence and the latency period was relatively low.

\section{CONCLUSION}

In conclusion, this study demonstrates the potential role of resveratrol in preventing and delaying the tumour development. In addition, it also causes down regulation of COX there by inhibiting transcription factors, modulating cell differentiation, proliferation and apoptosis at higher doses remarkably compared to suboptimal dose. Although there is further need for analyzing the pharmacogenetics and pharmacokinetics of resveratrol in breast cancer subjects. Further studies are required to establish the link between nuclear transcription factors and resveratrol.

\section{Funding: No funding sources \\ Conflict of interest: None declared}

Ethical approval: The study was approved by the Institutional Ethics Committee

\section{REFERENCES}

1. Waladkhani A, Clemens M. Effect of dietary phytochemicals on cancer development. Bioorg. Med. Chem. Lett. 1998;1:747-53.

2. Kelloff GJ, Crowell JA, Steele VE, Lubet RA, Malone WA, Boone CW, et al. Progress in cancer chemoprevention: development of diet-derived chemopreventive agents. J. Nutr. 2000;130:467-71. 
3. Howitz K, Bitterman K, Cohen H. Small molecule activators of sirtuins extend Saccharomyces cerevisiae life span. Nature. 2003;425:191-96.

4. Gehm BD, McAndrews JM, Chien PY, Jameson JL. Resveratrol, a polyphenolic compound found in grapes and wine, is an agonist for the estrogen receptor. Proc. Natl. Acad. Sci. USA. 1997;94:14138-43.

5. Jang M, Cai L, Udeani GO, Slowing KV, Thomas $\mathrm{CF}$, Beecher $\mathrm{CW}$, et al. Cancer chemo preventive activity of resveratrol, a natural product derived from grapes. Science. 1997;275:218-20.

6. Athar M, Back JH, Tang X, Kim KH, Kopelovich L, Bickers DR, et al. Resveratrol: a review of preclinical studies for human cancer prevention. Toxicol Appl Pharmacol. 2007;224:274-83.

7. Shankar S, Singh G, Srivastava R. Chemoprevention by resveratrol: molecular mechanisms and therapeutic potentials. Front Biosci. 2007;12:483954.

8. Holme A, Pervaiz S. Resveratrolin cell fate decisions. J Bioenerg Biomembr. 2007;39:59-63.

9. King R, Bomser J, Min D. Bioactivity of resveratrol. Compreh Rev Food Sci Food Safety. 2006;5:65-70.

10. Whitsett $\mathrm{T}$, Carpenter $\mathrm{M}$, Lamartiniere CA. Resveratrol, but not EGCG, in the diet suppresses DMBA-induced mammary cancer in rats. J Carcinog. 2006;5:1

11. De Martin R, Schmid J, Hofer R. The NF-B/Rel family of transcription factors in oncogenic transformation and apoptosis. Mutat. Res. 1999;437:231-43.

12. Gilmore TD, Koedood M, Piffat KA, White DW. Rel/NF-B/IB proteins and cancer. Oncogene. 1996;13:1367-78.

13. Jang M, Pezzuto J. Effects of resveratrol on 12-Otetradecanoylphorbol-13-acetate-induced oxidative events and gene expression in mouse skin. Cancer Lett. 1998;134:81-9.

14. Banerjee S, Ramos C, Aggarwal B. Suppression of 7,12-dimethylbenz(a)anthracene-induced mammary carcinogenesis in rats by resveratrol: role of nuclear factor-kappa B, cyclooxygenase 2, and matrix metalloprotease 9. Cancer Res. 2002;62:4945-54.

15. Jang M, Pezzuto J. Cancer chemo preventive activity of resveratrol. Drugs Exp. Clin. Res. 1999;25:65-77.

16. Hilakivi L. Estrogens, BRCA1 and breast cancer. Cancer Res. 2000;60:4993-01.

17. Lu R, Serrero G. Resveratrol, a natural product derived from grape, exhibits antiestrogenic activity and inhibits the growth of human breast cancer cells. J. Cell. Physiol. 1999;179:297-04.

Cite this article as: Suresh A, Rana R, Keerthana C. Resveratrol chemoprotective effect and COX 2 down regulation in tumor suppression in DMBA induced breast cancer in female Sprague Dawley rats. Int J Basic Clin Pharmacol 2017;6:1517-21. 